\title{
Use of Xpert Contributes to Accurate Diagnosis, Timely Initiation, and Rational Use of Anti-TB Treatment Among Childhood Tuberculosis Cases in South Central Ethiopia
}

This article was published in the following Dove Press journal:

Pediatric Health, Medicine and Therapeutics

\author{
Abebe Sorsa' \\ Degu Jerene $\mathbb{D}^{2}$ \\ Solomon Negash ${ }^{2}$ \\ Ashenafi Habtamu (D) \\ 'Department of Pediatric and Child \\ Health, Arsi University, College of Health \\ Sciences, Asella, Ethiopia; ${ }^{2}$ Management \\ Sciences for Health, USAID/Challenge TB \\ Project, Addis Ababa, Ethiopia
}

Background: Childhood tuberculosis (TB) was under-prioritized, and only $15 \%$ of childhood TB cases are microbiologically confirmed. Hence, most childhood TB diagnoses are made on a clinical basis and prone to over- or under-treatment. Xpert is a rapid method for the diagnosis of childhood TB with high sensitivity.

Objective: To assess the use of Xpert for accurate diagnosis, timely initiation, and rational use of anti-TB treatment among childhood TB.

Methods: In 2016, the hospital facilitated the installation of the Xpert machine. We reviewed data trends over four consecutive years; two years before the arrival of the machine and two years following the implementation of Xpert. Data were extracted retrospectively from electronically stored databases and medical records and entered to SPSS 21 for analysis.

Results: In the pre-intervention period (2014-2015), 404 cases of children presenting with symptoms or signs suggestive of TB ("presumptive TB") were evaluated using AFB microscopy. A total of $254(62.8 \%)$ TB diagnoses were made, of which 54 (21.3\%) were confirmed by smear AFB while 200 (78.7\%) were treated as smear-negative TB cases. The mean waiting time to start anti-TB treatment was 6.95 days [95\% CI (3.71-10.90)]. During the intervention period (2016-2017), 371 children with presumptive TB were evaluated using Xpert. A total of 199 (53.6\%) childhood TB cases were notified, of which 88 (44.2\%) were Xpert positive and $111(55.8 \%)$ were treated as Xpert-negative probable TB cases. The tendency to initiate anti-TB treatment for unconfirmed TB cases was reduced by a third. Compared with smear AFB, Xpert improved accuracy of diagnosing pediatric TB cases two-fold. The average waiting time to start anti-TB treatment was 1.33 days [ $95 \% \mathrm{CI}(0.95-1.71)$ ]. There was a significant reduction in the waiting time to start anti-TB treatment, with a mean time difference before and during intervention of 5.62 days [95\% CI (1.68-9.56)].

Conclusion: Xpert use was associated with a significant increase in the accuracy of identifying confirmed TB cases, reduced unnecessary anti-TB prescription, and shortened the time taken to start TB treatment.

Keywords: Xpert, AFB smear, childhood TB, confirmed TB, presumptive TB

\section{Background}

Childhood TB remains among the most prevalent infectious diseases resulting in significant morbidity and mortality in children. In settings where TB has a high burden, childhood TB accounts for $15-20 \%$ of total TB incidence. ${ }^{3}$ TB is one of the 
most contagious infectious human diseases with a high infection rate particularly in children aged less than five years. $^{1-3}$

TB is a poverty-related disease, disproportionately affecting the poorest, most vulnerable, and marginalized population groups wherever it occurs. Children with the disease fre-

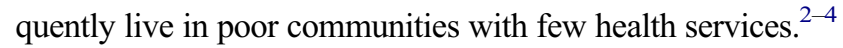

Ethiopia has been identified as one of the 30 High Burden Countries (HBC) for TB, TB and human immunodeficiency virus coinfection (TB/HIV), and multidrugresistant TB (MDR-TB). In 2016, Ethiopia had an annual estimated TB incidence of 177 people per 100,000 population and a death rate of 25 per 100,000 population. Among the notified TB cases in 2016, 2.7\% of new TB cases and $14 \%$ of previously treated TB cases were also estimated to harbor drug-resistant TB. ${ }^{4}$

Microscopy was the mainstay modality of TB diagnosis for years despite exhibiting low diagnostic sensitivity, particularly among children. Under optimal conditions, the sensitivity of smear microscopy for the diagnosis of childhood TB remains less than 15\%. Microbiological confirmation of mycobacterium TB by culture detected only $15-50 \%$ of pediatric cases and the procedure takes a longer time so it is not usually used for routine clinical care. Therefore, one of the 10 priority indicators for monitoring the implementation of the WHO End TB Strategy is the percentage of new and relapsing TB cases that have been tested with a WHO-recommended rapid diagnostic measure at the time of diagnosis. ${ }^{5-12}$

The Xpert system was introduced in 2004. It provides user-friendly molecular testing by fully integrating and automating the three processes required for real-time polymerase chain reaction (PCR)-based molecular testing (namely specimen preparation, amplification, and detection) and additionally detects both live and dead bacteria. ${ }^{8}$ Xpert represents a paradigm shift in the diagnosis of TB and simultaneously detecting multidrug resistance. It amplifies sputum samples in a closed system suitable for use outside conventional laboratory settings. The turnaround of Xpert is less than 2 hours. 9,10

The sensitivity of the test is much better than smear microscopy and similar to that of solid culture. The limit of detection is 5 genome copies of purified DNA per reaction or 131 colony-forming units/mL in mycobacterium TB whereas identification of TB bacilli by microscopic examination requires at least 10,000 bacilli per milliliter of sputum. ${ }^{9-11}$ Upfront access to Xpert testing in pediatric presumptive pulmonary TB cases was associated with a two-fold increase in bacteriologically confirmed pulmonary TB. ${ }^{4}$

In the current analysis, we examined childhood presumptive TB data collected before and after the implementation of Xpert. Our goal was to assess the significance of the improvement afforded by Xpert for TB diagnosis, use of anti-TB treatment for unconfirmed cases, and waiting time to initiate anti-TB treatment among positive cases.

\section{Materials and Methods Study Setting, Design, and Selection of Study Units}

We conducted a retrospective medical document review study which was approved by the Arsi University institutional review board. The study was conducted in 2018 at Asella Teaching and Referral Hospital (ATRH), located about $175 \mathrm{~km}$ from the capital, Addis Ababa. The hospital is the only teaching and referral center in the region of South-Central Ethiopia delivering service to a population of approximately four million.

Acid-Fast Bacillus (AFB) smear microscopy has been used for the clinical diagnosis of TB at ATRH for decades. In the beginning of 2016, the Xpert machine was installed by the minister of health in collaboration with Challenge TB Ethiopia. The hospital was selected as a pilot site and the Xpert service was indicated to childhood TB and TBHIV co-infection. All relevant health professionals attended on-site training on the operation of the machine and samples were processed using a standard operating procedure. We enrolled all cases of childhood presumptive TB (<15 years) from January 1, 2014, to December 31, 2017, who met the criteria for childhood presumptive TB; based on two or more typical symptoms of TB (cough, fever, poor weight gain) for more than 2 weeks or contact with a patient diagnosed with TB.

\section{Data Collection and Management}

All childhood presumptive TB cases during the consecutive four years (2014-2017) were included. Data were sourced from patient medical records, hospital electronic medical records, and the TB clinic registry book. Laboratory results were retrieved from the laboratory center data storage unit. Consistency, completeness, and accessibility of the data were assessed using a random subset of the data. For each variable reported in this study, approximately 20 medical records and digitally stored database entries were randomly reviewed to ensure that the objectives of the study were met. 
Data collectors were blinded to the results of each case. Data collectors were nurses qualified in chart review and underwent a two-day training course on the use of the data collection tool. The data collectors independently reviewed clinical notes and completed a standardized dataentry format for presenting symptoms, time taken to receive laboratory result, final diagnosis, and date of starting anti-TB treatment for positive cases. Data collectors were supervised and could ask advice concerning any ambiguities or other problems. All data were reviewed by a third person for consistency and completeness. The primary outcome was the proportion of patients to receive a positive TB diagnosis.

\section{Data Analysis}

Descriptive statistics and cross-tabulation were used to quantitatively analyze the relationship between data acquired for patients before and after the implementation of the new diagnostic technology. A paired $T$-test was used to estimate the difference in waiting time before the commencement of anti-TB treatment in the two time periods. Adjusted odds ratios were calculated for factors associated with the effect of Xpert and smear AFB using a multiple logistic regression.

\section{Operational Definitions $2,3,7,9,18,21$}

Presumptive TB: child presented with a cough lasting two or more weeks AND/OR any of the following clinical symptoms: contact with a TB patient, fever, weight loss, or failure to gain weight.

Confirmed TB: child with at least 1 defined sign or symptom suggestive of TB AND microbiologically confirmed TB, defined as at least one positive smear or positive Xpert.

Probable TB: child meets two or more of the following criteria: history of TB contact, a clinical feature suggestive of $\mathrm{TB}$, reactive tuberculin, skin test $>10 \mathrm{~mm}$, or radiographic findings compatible with TB (miliary TB, cavitary lesions, hilar lymphadenopathy, or primary complex), no response to trial of broad-spectrum antibiotics.

Compatible Chest-X-ray: Hilar lymphadenopathy, cavitary lesion (common with older children), and miliary TB.

Trial of broad-spectrum antibiotics: antibiotic treatment prescribed for a patient with presumptive TB who has been evaluated clinically, radiologically, and microbiologically, in which the findings were inconsistent with TB and therefore the patient was given broad-spectrum antibiotics for a possible alternative diagnosis of pneumonia.

\section{Ethical Considerations}

Ethical clearance was obtained from Arsi University, College of Health Sciences research ethical review board. As per the Arsi University college of health science ethical review board, consent of patient/guardian is not needed for secondary data research as it is not feasible to access them. Hence, the requirement for obtaining consent was waived and the research was conducted in accordance with the Declaration of Helsinki. However, confidentiality of information retrieved from patients' medical records was upheld and data were not disclosed to any third parties.

\section{Results}

A total of 775 presumptive TB cases with complete medical records were enrolled; 404 of these formed the preintervention group (January 2014-December 2015), and the remaining 371 subsequent to the installation of the Xpert system (January2016-December2017). Over the four-year period, a total of $453(58.5 \%)$ patients were diagnosed with TB. From the total TB cases, 142 $(31.3 \%)$ were confirmed microbiologically either using AFB microscopy or Xpert, and 311 (68.7\%) were microbiological negative and treated as "probable" TB cases (see Table 1).

\section{Subgroup Analysis \\ Pre-Intervention (2014-2015)}

A total of $404(n=204$ male; 50.1\%) childhood presumptive TB cases were evaluated using smear AFB. Cough, fever, and weight loss were the leading clinical presentations (see Table 1). The mainstay of TB diagnosis was conventional AFB microscopy. A total of 54 (13.4\%) were found to be smear AFB positive. During this period, a total of 254 childhood TB diagnoses were made, of which 54 (21.3\%) were confirmed by AFB microscopy and 200 $(78.7 \%)$ were treated as smear-negative "probable" TB cases. A total of $244(60.4 \%)$ cases were prescribed a trial of broad-spectrum antibiotics for a possible alternative diagnosis other than that of a bacterial respiratory infection. Of these, 105 (43.0\%) made a clinical improvement, and the remaining $139(57.0 \%)$ were re-enrolled for TB work up. There was no association between antibiotic use and the rate of positive smear AFB, AOR 2.69 (95\% CI, 082-8.30). The smear-positive rate was comparable in children under five years of age and those between 5 and 15 years (see Table 2). 
Table I Patients Clinical Feature and Some Laboratory Characteristics, ATRH, 2018

\begin{tabular}{|c|c|c|c|c|}
\hline \multicolumn{2}{|l|}{ Demographic } & \multirow{3}{*}{$\begin{array}{l}\text { Pre-Intervention, } n=404 \\
10(7-12) \\
204(50.1) \\
200(49.9)\end{array}$} & \multirow{3}{*}{$\begin{array}{l}\text { During Intervention, } \mathbf{n = 3 7 |} \\
8(4-12) \\
199(53.6) \\
172(46.4)\end{array}$} & \multirow{3}{*}{$\begin{array}{l}\frac{p-v a l u e}{0.121} \\
0.065\end{array}$} \\
\hline Age & ), year & & & \\
\hline Sex & $\begin{array}{l}\text { Male } \\
\text { Female }\end{array}$ & & & \\
\hline \multicolumn{5}{|c|}{ Presenting clinical feature } \\
\hline \multicolumn{2}{|l|}{ Cough, n(\%) } & $375(91.9)$ & $352(94.9)$ & 0.056 \\
\hline \multicolumn{2}{|l|}{ Fever, $n(\%)$} & $315(77.2)$ & $277(74.7)$ & 0.072 \\
\hline \multicolumn{2}{|l|}{ Weight loss, n(\%) } & $250(61.3)$ & $242(65.2)$ & 0.062 \\
\hline \multicolumn{2}{|c|}{ Severe acute malnutrition, $\mathrm{n}(\%)$} & $5 I(12.5)$ & $49(13.2)$ & 0.323 \\
\hline \multicolumn{2}{|c|}{ BCG Vaccination, $\mathrm{n}(\%)$} & $4 I(10.0)$ & $62(16.7)$ & 0.051 \\
\hline \multicolumn{2}{|c|}{ Contact with chronic cougher, $n(\%)$} & $106(26.0)$ & $135(36.4)$ & 0.032 \\
\hline \multicolumn{2}{|c|}{ Contact with TB patients, $\mathrm{n}(\%)$} & $75(18.4))$ & $116(31.3)$ & 0.012 \\
\hline \multicolumn{2}{|c|}{ Trial of antibiotic use, $n(\%)$} & $244(60.0)$ & $247(66.6)$ & 0.071 \\
\hline \multicolumn{2}{|c|}{ Improved for trial of antibiotic, $n(\%)$} & $105(25.7)$ & $165(45.0)$ & 0.013 \\
\hline \multicolumn{2}{|c|}{ Highly elevated ESR(>50mm/hr) } & $210(51.5)$ & $|8|(48.8)$ & $0.08 \mathrm{I}$ \\
\hline \multicolumn{2}{|c|}{ Anemia(hemoglobin< $<\mid \mathrm{g} / \mathrm{dl})$} & $122(29.9)$ & $101(27.2)$ & 0.187 \\
\hline \multicolumn{2}{|c|}{$\begin{array}{l}\text { Waiting time to start anti-TB, } \\
\text { median (IQR), days }\end{array}$} & $3(2-5.75)$ & $2(0-4)$ & 0.015 \\
\hline
\end{tabular}

Abbreviations: ATRH, Asella Teaching and Referral Hospital; BCG, Bacille Calmette-Guérin; ESR, erythrocyte sedimentation rate; IQR, interquartile range; TB, tuberculosis.

Table 2 Multivariate Logistic Regression: Factors Associated with Tuberculosis Case Notification at ATRH, 2018

\begin{tabular}{|c|c|c|c|c|}
\hline & “Probable” TB Cases, N=3 I I & Smear AFB Positive $\mathbf{N}=54$ & XPert Positive $\mathbf{N}=\mathbf{8 8}$ \\
\hline & & AOR, $95 \% \mathrm{Cl}$ & AOR, $95 \% \mathrm{Cl}$ & AOR, $95 \% \mathrm{CI}$ \\
\hline BCG vaccine & No & $0.81(0.55-1.21)$ & $2.06(0.96-2.38)$ & $1.06(0.57-2.03)$ \\
\hline SAM & Yes & $3.29(I .9 I-5.7 I)$ & $1.97(0.87-4.44)$ & $2.00(1.07-3.70)$ \\
\hline Contact with chronic cougher & Yes & $1.56(1.14-2.15)$ & $1.28(0.66-2.47)$ & $1.55(0.96-2.53)$ \\
\hline Contact with TB patient & Yes & $1.67(1.24-2.24)$ & $1.03(0.56-1.88$ & $\mathrm{I} .44(0.89-2.31)$ \\
\hline Weight loss & Yes & $1.21(0.92-1.6 I)$ & $2.03(1.11-3.72)$ & $1.33(0.77-2.30)$ \\
\hline Age category & $\geq 5$ years & $1.73(1.25-2.40)$ & $1.01(0.45-2.55)$ & $1.02(0.59-1.75)$ \\
\hline Anemia & Yes & $1.81(1.38-2.38)$ & $2.08(1.21-3.58)$ & $1.22(0.76-1.95)$ \\
\hline \multirow[t]{2}{*}{ ESR level (mm/hr) } & $50-99$ & $2.06(1.46-2.91)$ & $1.95(0.83-4.61)$ & $2.42(1.24-4.73)$ \\
\hline & $\geq 100$ & $5.9(2.6-13.3)$ & $5.26(1.8-15.7)$ & $4.55(1.51-13.7)$ \\
\hline Chest $X$-ray compatible with TB & Yes & II.3(7.4-17.4) & $5.7(3.2-10.2)$ & $7.5(4.5-12.6)$ \\
\hline Xpert implementation & Before & $1.89(1.44-2.78)$ & & \\
\hline
\end{tabular}

Abbreviations: AFB, acid-fast bacilli; AOR, adjusted odds ratio; ATRH, Asella Teaching and Referral Hospital; BCG, Bacille Calmette-Guérin; Cl, confidence interval; ESR, erythrocyte sedimentation rate; SAM, severe acute malnutrition; TB, tuberculosis.

\section{During Xpert Use (2016-2017)}

Xpert was introduced as a primary diagnostic modality of TB for under 15 years of age. During this period, a total of 371 childhood presumptive TB cases were evaluated $(\mathrm{n}=$ 199 male; 53.6\%). Cough, fever, and weight loss were the leading clinical presentations (see Table 1). Of these cases, $88(23.7 \%)$ were positive for mycobacterium TB (MTB), and no cases of rifampicin resistance were reported. During this period, a total of 199 TB cases were diagnosed of which $88(44.2 \%)$ were confirmed by Xpert, and 111 
$(55.8 \%)$ were treated as "probable" TB cases. This represents a $77 \%$ (almost twofold) increase proportionately in the number of presumptive TB cases to be microbiologically confirmed from the first period to the second period.

A total of 247 (66.4\%) patients were prescribed a trial of broad-spectrum antibiotics for a possible alternative diagnosis of non-TB bacterial respiratory tract infection, of which 165 (45.0) showed a clinical response to antibiotic treatment. The proportion of patients prescribed with broad-spectrum antibiotic treatment is not significantly different between the two periods but the number of patients showing a clinical response to the antibiotics significantly increased following implementation of Xpert (Table 1 and Figure 1).

The waiting time for TB-positive patients to start anti-TB treatment was compared between the two periods. The implementation of Xpert significantly shortened the waiting time to an average of 2.6 days ( $95 \%$ CI $1.5-3.5 \mathrm{p} 0.014$ ) compared to 5.5 days (95\% CI 3.7-7.4) beforehand. Moreover, onequarter of TB patients diagnosed using Xpert started antiTB treatment on the day of evaluation compared to only $7.5 \%$ of TB patients in the first period (Figure 2).

\section{Factors Affecting the Rate of Positive Smear AFB vs Xpert}

Patients who had contact with a patient with TB or chronic cough were likely to have their TB diagnosis confirmed by either AFB microscopy or Xpert (odds ratio $1.57,95 \%$ CI 1.09-2.25, p-0.014).

Bacille Calmette-Guérin (BCG) vaccination status did not show any association with any form of TB diagnosis. Patients with chest $\mathrm{X}$-ray findings consistent with pulmonary $\mathrm{TB}$, elevated erythrocyte sedimentation rate (ESR), and presence of malnutrition were highly likely to have a positive smear AFB or Xpert result (Table 2).

The trends of confirmed TB cases increased after the introduction of Xpert while "probable" TB cases decreased in line with the overall trend in decreasing TB case presentation.

Prescription of broad-spectrum antibiotic treatment for respiratory complaints has significantly reduced after the introduction of Xpert compared the preceding two years (odds ratio $0.134,95 \%$ CI $0.08-0.23, \mathrm{p}<0.001)$. This may indicate improved rationale in the use of antibiotics (Figure 1). There was no association between antibiotic use and positive Xpert results (odds ratio $2.33,95 \% \mathrm{CI}, 0.49-11.10$ ).

Similarly, the initiation of anti-TB treatment for cases of unconfirmed TB decreased significantly during intervention.

\section{Discussion}

There is a critical need for improved diagnosis of TB in children, particularly in young children as this deficit represents the most neglected sectors of population and those posing the greatest diagnostic challenges. There is also

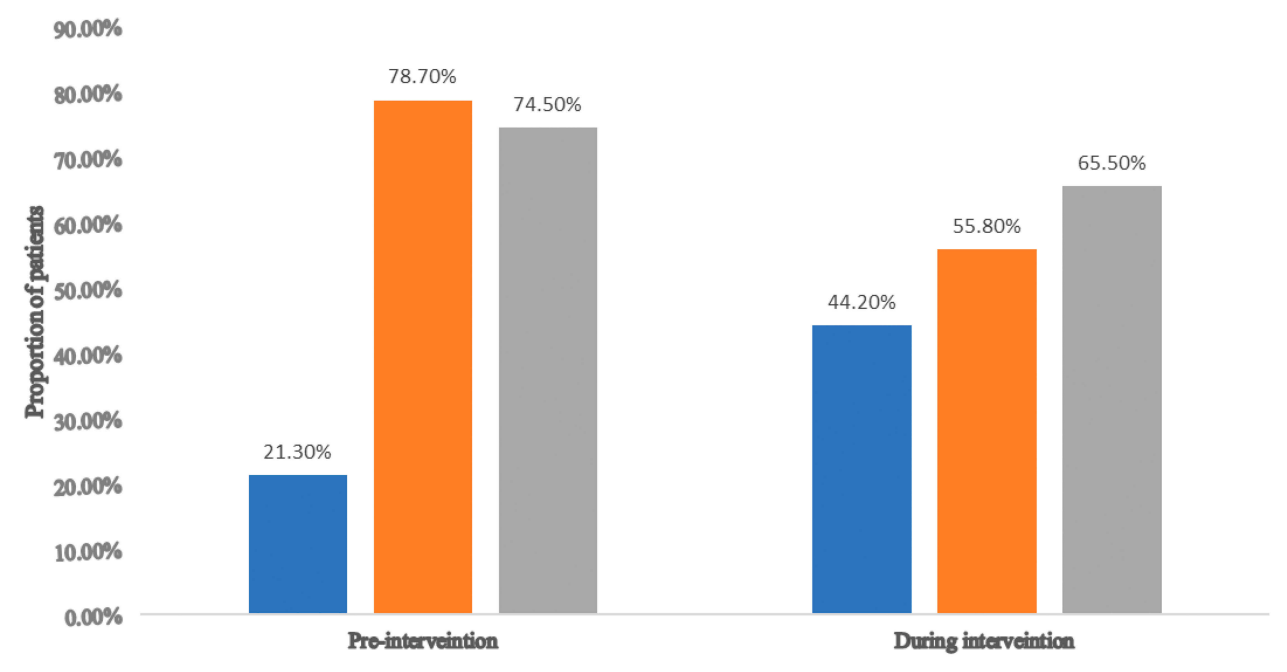

m Proportion of microbiologically confirmed TB cases $\|$ Probable or possible TB cases $\approx$ Broad spectrum antibiotic prescription

Figure I Bar chart comparison of trends of microbiologically confirmed TB cases, probable or possible TB cases, and utilization of antibiotics at Asella Teaching and Referral Hospital in 2018. 


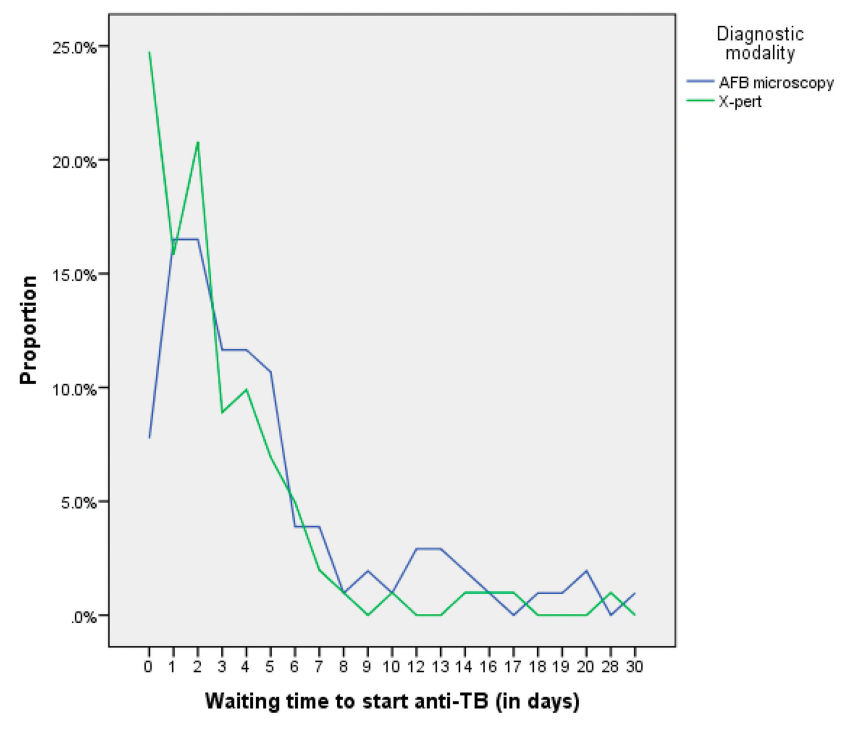

Figure 2 Statistical summary of trends of waiting time to start anti-TB before and during intervention at Asella Teaching and Referral Hospital in 2018.

a need for standardization of clinical definitions for the evaluation of diagnostic methods in prospective clinical research studies, including those involving children in whom TB is suspected but not confirmed microbiologically., ${ }^{2,6}$ The present study found that prior to the implementation of Xpert, the rate of positive smear AFB results amongst presumptive TB cases was 54/404 (13.4\%). Of cases to receive a positive diagnosis, $54 / 254(21.3 \%)$ were microbiologically confirmed, making the remaining 200 cases $(78.7 \%)$ were treated as smearnegative "probable" TB diagnoses. This finding is consistent with the findings of a national survey, which reported the proportion of smear-positive TB cases to be $23.1 \%$ with threequarters of patients being treated for unconfirmed TB diagnoses. ${ }^{2,4,10}$ This diagnostic disparity motivated the recent introduction of the new diagnostic modality known as Xpert.

In our study, the proportion of total presumptive TB cases to receive a positive diagnosis of MTB using Xpert was found to be $88 / 371(23.7 \%)$, an almost two-fold increase compared to using smear AFB. This finding is higher than a recent study from France which reported smear microscopy and gene Xpert detection rates to be $4.8 \%$ and $10.4 \%$, respectively. This difference could be explained by the difference in the epidemiological distribution of the disease between the two countries, as well as differences in the definitions of suspected TB and the skill and experience levels of individuals performing AFB microscopy. ${ }^{4}$

In this study, the diagnostic efficacy of Xpert MTB was assessed in comparison to conventional AFB smear microscopy. Taking the clinical definition of TB diagnosis as the gold standard, Xpert showed improved diagnostic capacity compared to AFB smear microscopy, demonstrated through a two-fold increase in the number of confirmed TB diagnoses using Xpert compared to AFB smear microscopy. This is in agreement with reports from South Africa and Brazil showing increases in confirmed TB diagnoses of $30-37 \% .^{1,2,5-7}$ In our study, unconfirmed TB diagnoses decreased significantly after the introduction of Xpert. The decline in "probable" TB cases' after the introduction of Xpert reduced the margin of error on the decision to commence anti-TB treatment. As such, this mitigates the negative impact of unnecessary anti-TB treatment on a significant number of patients and their families., 3,4,8-10 Most patients who were initially presumed to have TB and who received a negative Xpert diagnosis were given an alternative diagnosis and improved with the prescription of broad-spectrum antibiotics. Broad-spectrum antibiotic use was not statistically different before and after implementation of Xpert; however, the number of patients showing a positive response to prescribed antibiotics was significantly higher in the latter period. This is likely due to the higher sensitivity and specificity of Xpert compared to AFB microscopy. A study by Lucian Davis et al and others reported that continued Xpert use could conceivably have significant clinical and public health consequences by decreasing unnecessary use of anti-TB treatment through contact screening. Pulmonary TB was the most commonly diagnosed form of TB in the present study, which is in agreement with 2019 WHO survey report among other reports where the majority of cases were diagnosed in children older than 5 years of age. ${ }^{12-17,25}$

The waiting time between first hospital visit and commencement of anti-TB treatment is one of the critical priorities for interrupting disease transmission and halting the circulation of the bacilli in the community. It is also equally important to start the patient on treatment rapidly so as to decrease patient suffering and improve clinical outcome. $^{26,30}$ In line with this, our study assessed the impact of Xpert in shortening waiting times. We reported that the implementation of Xpert significantly reduced the average waiting time to start anti-TB treatment, indicating reducing patient suffering, improved treatment outcomes, reduced risk of transmission, and improved TB control overall. A recent multicenter study showed that the median time to positive TB diagnosis for the gene Xpert test was zero (0) days (IQR 0-1), compared with 1 day (0-1) for microscopy, 30 days (23-43) for solid culture, and 16 days (13-21) for liquid culture. Use of Xpert reduced the 
median time to treatment for smear-negative TB from 56 days (39-81) to 5 days. ${ }^{6,7,9,11,14}$

Children who received BCG vaccination were at lower risk of having smear-positive TB while its overall TB preventive efficacy is not significantly different from the unvaccinated population. BCG vaccination reduces severe forms of mycobacterium TB infection (eg, central nervous system TB) in children; however, it has only minimal effects on secondary TB and TB of the lung. BCG, therefore, plays an insignificant role in strategies to control TB. ${ }^{2,3,17}$

Our study demonstrated that the implementation of Xpert considerably decreased the frequency and impact of unnecessary broad-spectrum antibiotic prescription for presumptive TB cases which is in agreement with the findings of other studies. Similarly, even though the present study did not entirely characterize the impact of Xpert in contact case tracing, there are a number of reports indicating that its use can also improve contact screening. ${ }^{1-3,4,19-24,27-30}$

\section{Limitations}

The research findings were generated by using retrospective data analysis through reviewing patient medical records. Data retrieval was subject to some issues of inconsistency and incompleteness. However, the output of the research carries a significant scientific impact and provides a fertile ground for future prospective studies.

\section{Conclusion and Recommendation}

The implementation of Xpert was associated with a significant increase in the proportion of confirmed childhood TB cases and a significant reduction in prescription of anti-TB treatment for unconfirmed TB cases and improved response to the use of antibiotics for patients receiving a TB-negative diagnosis. Further, Xpert use was associated with a significant decrease in the time taken to start anti-TB treatment among children presenting as presumptive TB. Xpert use provides significant improvement in the TB diagnosis and treatment of children. With widerscale use, it will have a profound impact on the success of the WHO End TB Strategy.

\section{Abbreviations}

AFB, Acid-Fast Bacilli; AIDS, Acquired Immune Deficiency Syndrome; ATRH, Asella Teaching and Referral Hospital; BCG, Bacille Calmette-Guérin; EPHI, Ethiopian Public Health Institute; ESR, Erythrocyte Sedimentation Rate; FMOH, Federal Ministry of Health;
HIV, Human Immunodeficiency Virus; MDR-TB, MultiDrug Resistance Tuberculosis; TB, Tuberculosis; MTB/ RIF, Macrobacterium Tuberculosis/Rifampicin; PCR, Polymerase Chain Reaction.

\section{Acknowledgments}

We are very much grateful for the health professionals working at Asella Teaching and Referral Hospital, pediatric and child health department, for their unreserved cooperation and commitment during data collection. Next, we are equally grateful to Rebecca Dewey for her extensive and constructive English language editing support to finalize the manuscript.

\section{Disclosure}

The authors report no conflicts of interest in this work.

\section{References}

1. Stephen MG, Tahmeed A, Farhana A, Renee B. Evaluation of tuberculosis diagnostics in children: proposed clinical case definitions for classification of intrathoracic tuberculosis disease. Consensus from an expert panel. $J$ Infect Dis. 2012;205(1).

2. Guillermo ES, Schmitz TL, Cama R, et al. Pulmonary tuberculosis in children in a developing country. Pediatrics. 2001;108(2). doi:10.1542/peds.108.6.1367

3. Qing QY, Wei WJ, Rui H. Rapid diagnosis of childhood pulmonary tuberculosis by Xpert MTB/RIF assay using bronchoalveolar lavage fluid. BioMed Res Int. 2014;2014:310194

4. Neeraj R, Kuldeep Singh S, Sreenivas AN, et al. Enhancing TB case detection: experience in offering upfront Xpert MTB/RIF testing to pediatric presumptive TB and DR TB cases for early rapid diagnosis of drug sensitive and drug resistant TB. PLoS One. 2014;9(8): e105346.

5. Durovni B, Saraceni V, van den Hof S, et al. Impact of replacing smear microscopy with Xpert MTB/RIF for diagnosing tuberculosis in Brazil: a stepped-wedge cluster-randomized trial. PLoS Med. 2014;12(12):e1001928.

6. Dodd J, Gardiner E, Coghlan R, Seddon A. Burden of childhood tuberculosis in 22 high-burden countries: a mathematical modeling study. Lancet Glob Health. 2014;2:453-459. doi:10.1016/S2214109X(14)70245-1

7. Federal Democratic Republic of Ethiopia Minister of Health Guidelines for Clinical and Programmatic Management of TB, Leprosy and TB/HIV in Ethiopia. 6th ed. 2018.

8. World Health Organization. Global Tuberculosis Report; 2019

9. Federal Democratic Republic of Ethiopia Minister of Health. National Population Based Tuberculosis Prevalence Survey. Ethiopia Health and Nutrition Research Institute; 2015.

10. Berti E, Galli L, Venturini E, et al. Tuberculosis in childhood: a systematic review of national and international guidelines. $B M C$ Infect Dis. 2014;14(1):S3.

11. World Health Organization. Multidrug-Resistant Tuberculosis (MDRTB); 2016.

12. David P, Ellen J, Fred T. The Need for Better Diagnostic Tests for Pediatric Tuberculosis. Vol. 4. 2011:3-7.

13. Claude K. Paediatric Tuberculosis: Is the World Doing Enough? World's Largest Science, Technology \& Medicine. Open Access book publisher; 2013:372-380. 
14. World Health Organization. Xpert MTB/RIF Implementation Manual; 2014.

15. Theron G, Peter J, van Zyl-smit R. Evaluation of the Xpert MTB/RIF assay for the diagnosis of pulmonary tuberculosis in a high HIV prevalence setting. Am J Respir Crit Care Med. 2011;184 (1):132-140. doi:10.1164/rccm.201101-0056OC

16. Haider A, Abed A, Humaira A, Kee P, Frederick L. The diagnostic performance of a single geneXpert MTB/RIF assay in an intensified tuberculosis case. PLoS One. 2013;8(9).

17. Jain SK, Ordonez A, Kinikar A, et al. Pediatric tuberculosis in young children in India: a prospective study. Biomed Res Int. 2013;2013:783698.

18. Richard J. Impact of the Xpert MTB/RIF tuberculosis diagnostic system in individuals at high risk of mortality in rural South Africa. London School Hygiene \& Tropical Medicine; 2015.

19. Zeka AN, Sezai T, Cengiz C. Evaluation of the GeneXpert MTB/RIF assay for rapid diagnosis of tuberculosis and detection of rifampin resistance in pulmonary and extra pulmonary specimens. $J$ Clin Microbiol. 2011;49(12):4138-4141. doi:10.1128/JCM.05434-11

20. Do C, Tran Ngoc D, Dang T, Ho T, Marcel W. Prospective evaluation of GeneXpert for the diagnosis of HIV-negative pediatric TB cases. BMC Infect Dis. 2015;15(70).

21. Davis JL, Masae Kawamura L, Chaisson LH, Jennifer G. Impact of GeneXpert MTB/RIF on patients and tuberculosis programs in a low-burden setting. Am J Respir Crit Care Med. 2014;189 (12):1551-1559. doi:10.1164/rccm.201311-1974OC

22. Evans CA. Gene Xpert-A game changer for Tuberculosis control. PLoS Med. 2011;8(7):e1001064. doi:10.1371/journal.pmed.1001064

23. Menzies NA, Ted C. Population health impact and cost effectiveness of tuberculosis diagnosis with Xpert MTB/RIF: dynamic simulation and economic evaluation. PLoS Med. 2012;9(11). doi:10.1371/journal.pmed.1001347
24. Catharina C, Mark B, Pamela N, Joy M. Feasibility, diagnostic accuracy, and effectiveness of decentralized use of the Xpert MTB/ RIF test for the diagnosis of tuberculosis and multi drug resistance: a multi-center implementation study. Lancet. 2011;377:1495-1505. doi:10.1016/S0140-6736(11)60438-8

25. Gesine M, Kathryn S, Lawrence L, William M. The impact and cost of scaling up GeneXpert MTB/RIF in South Africa. PLoS One. 2015;7(5):e36966

26. Laura M, Natasha L, Jurgens AP, Stephen D. Diagnostic accuracy of the Xpert MTB/RIF assay for extra pulmonary and pulmonary tuberculosis when testing non-respiratory samples: a systematic review. BMC Infect Dis. 2014;14(709).

27. Habte D, Melese M, Hiruy N, Gashu Z. The additional yield of GeneXpert MTB/RIF test in the diagnosis of pulmonary tuberculosis among household contacts of smear positive TB cases. Int J Infect Dis. 2016;49:179-184. doi:10.1016/j.ijid.2016.07.002

28. Grant T, Jonny P, Greg C, Richard M. Determinants of PCR performance (Xpert MTB/RIF), including bacterial load and inhibition, for TB diagnosis using specimens from different body compartments. Sci Rep. 2014;4: 5658.

29. Monika A, ShiSh B, Vinay B. Comparative study of GeneXpert with $\mathrm{ZN}$ stain and culture in samples of suspected pulmonary tuberculosis. $J$ Clin Diagn Res. 2016;10(5):DC09-12.

30. Jenkins HE. Global burden of childhood tuberculosis. BMC Jenkins Pneumonia. 2016;8(1):24.
Pediatric Health, Medicine and Therapeutics

\section{Publish your work in this journal}

Pediatric Health, Medicine and Therapeutics is an international, peerreviewed, open access journal publishing original research, reports, editorials, reviews and commentaries. All aspects of health maintenance, preventative measures and disease treatment interventions are addressed within the journal. Practitioners from all disciplines are invited to submit their work as well as healthcare researchers and patient support groups. The manuscript management system is completely online and includes a very quick and fair peer-review system. Visit http://www.dovepress.com/testimonials.php to read real quotes from published authors. 